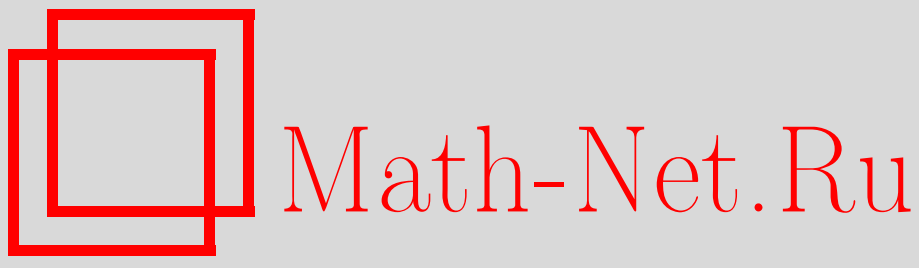

Л. Б. Голинский, И. Е. Егорова, О предельных множествах для дискретного спектра комплексных якобиевых матриц, Матем. сб., 2005, том 196, номер 6, 43-70

DOI: https://doi.org/10.4213/sm1364

Использование Общероссийского математического портала Math-Net.Ru подразумевает, что вы прочитали и согласны с пользовательским соглашением

http://www . mathnet.ru/rus/agreement

Параметры загрузки:

IP: 54.224 .187 .69

26 апреля 2023 г., $11: 39: 14$ 
УДК 517.5

\author{
Л. Б. Голинский, И.Е. Егорова
}

\title{
О предельных множествах для дискретного спектра комплексных якобиевых матриц
}

\begin{abstract}
Изучается дискретный спектр комплексных якобиевых матриц, которые являются компактнњми возмущениями дискретного лапласиана. Найдена точная в смысле порядка скорость стабилизации матричных элементов, при которой дискретный спектр конечен. Построен пример якобиевой матрицы, дискретнњй спектр которой имеет единственную предельную точку. Эти результаты представляют собой дискретные аналоги известных теорем Б. С. Павлова об операторах Шрёдингера на полуоси с комплексньп потенциалом.
\end{abstract}

Библиография: 26 названий.

\section{§1. Введение}

В 1960-х годах Б. С. Павлов, изучая дискретный спектр оператора Шрёдингера на полуоси

$$
l_{h} y=-y^{\prime \prime}+q(x) y, \quad y^{\prime}(0)-h y(0)=0,
$$

с комплекснозначным потенциалом $q$ и комплексным параметром $h$, пришел к следующим результатам [1], [2].

ТЕОРема П1. Число собственных значений оператора $l_{h}$ конечно, если бесконечно дифференцируемый потенциал $q(x)$ удовлетворяет при некотором $\varepsilon>0$ условию

$$
|q(x)| \leqslant C \exp \left(-\varepsilon x^{1 / 2}\right), \quad x>0 .
$$

ТЕОрема П2. Каковы бы ни были числа $0<\beta<1 / 2$ и $\lambda>0$ существует оператор ШІрёдингера (1.1) с вещественным бесконечно дифференцируемым потенциалом $q(x)$, удовлетворяющим при некотором $\delta>0$ условию

$$
|q(x)| \leqslant C \exp \left(-\delta x^{\beta}\right), \quad x>0,
$$

который при некотором комплексном $h$ имеет бесконечное множество невещественных собственных значений с единственной точкой накопления $\lambda$.

Целью настоящей работы является получение дискретных аналогов теорем Павлова.

Работа вьполнена при поддержке фонда INTAS Research Network NeCCA (грант № 0351-6637).

(C) л.Б. Голинский, И. Е. ЕгоровА 2005 
Пусть

$$
J=\left(\begin{array}{ccccc}
b_{0} & c_{0} & & & \\
a_{0} & b_{1} & c_{1} & & \\
& a_{1} & b_{2} & c_{2} & \\
& & \ddots & \ddots & \ddots
\end{array}\right)
$$

- бесконечная якобиева матрица с комплексными элементами, причем

$$
\lim _{n \rightarrow \infty} a_{n}=\lim _{n \rightarrow \infty} c_{n}=\frac{1}{2}, \quad \lim _{n \rightarrow \infty} b_{n}=0 .
$$

Оператор $J$ в пространстве $\ell^{2}\left(\mathbb{Z}_{+}\right), \mathbb{Z}_{+}=\{0,1,2, \ldots\}$, порождаемьй данной матрицей, является, тем самым, компактным возмушением дискретного лапласиана

$$
J_{0}: \quad a_{n}=c_{n}=\frac{1}{2}, \quad b_{n}=0 .
$$

Согласно теореме Г. Вейля спектр $\sigma(J)$ оператора $J$ есть $\sigma(J)=[-1,1] \cup \sigma_{d}(J)$, где не более чем счетное множество $\sigma_{d}(J)$ собственных значений конечной алгебраической кратности, называемое далее дискретным спектром оператора $J$, может иметь точки сгушения лишш на отрезке $[-1,1]$. Основньм объектом исследования является предельное множество дискретного спектра $E_{J}:=\left\{\sigma_{d}(J)\right\}^{\prime}$ для операторов (1.2), (1.3).

Возникает естественный вопрос о внутреннем описании класса $\left\{E_{J}\right\}$ замкнутых множеств на $[-1,1]$, которые могут быть предельными множествами дискретного спектра якобиевых матриц всего класса (1.2), (1.3) или некоторых его подклассов. Наши результаты представляют собой первый шаг на пути решения этого обшего вопроса, относящийся в основном к случаю пустого предельного множества (т.е. конечного дискретного спектра).

Будем говорить, что матрица $J(1.2)$ принадлежит классу $\mathscr{P}(\beta)$, если

$$
\left|a_{n}-\frac{1}{2}\right|+\left|c_{n}-\frac{1}{2}\right|+\left|b_{n}\right| \leqslant C_{1} \exp \left(-C_{2} n^{\beta}\right), \quad 0<\beta<1, \quad C_{1}, C_{2}>0 .
$$

Напомним, что показателем сходимости или индексом Тейлора-Безиковича замкнутого множества $F \subset[-1,1]$ называется величина

$$
\tau(F):=\inf \left\{\varepsilon>0: \sum_{j=1}^{\infty}\left|l_{j}\right|^{\varepsilon}<\infty\right\}
$$

где $\left\{l_{j}\right\}$ - смежные интервалы множества $F$.

Tеорема 1. Пусть $J \in \mathscr{P}(\beta)$, где $0<\beta<1 / 2$. Тогда $E_{J}-$ замкнутое множество меры нуль и его показатель сходимости удовлетворяет неравенству

$$
\operatorname{dim} E_{J} \leqslant \tau\left(E_{J}\right) \leqslant \frac{1-2 \beta}{1-\beta},
$$

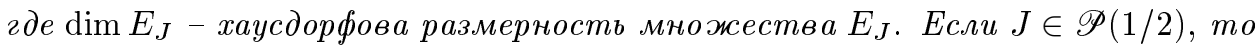
$E_{J}=\varnothing$ (т.е. дискретный спектр конечен).

Утверждение, касаюшееся конечности дискретного спектра, получено в несколько более обшем виде в работе [3; теорема 4.6].

Оказывается, показатель $1 / 2$ в теореме 1 является точным в следуюшем смысле. 
Tеорема 2. Для любъх $\varepsilon>0 u-1<\nu<1$ существует оператор $J \in$ $\mathscr{P}(1 / 2-\varepsilon)$ такой, что его дискретный спектр $\sigma_{d}(J)$ бесконечен и, более того, множество $E_{J}$ состоит из единственной точки $E_{J}=\{\nu\}$.

Отметим, что для вещественных якобиевых матриц $J$ с $b_{n}=\overline{b_{n}}, a_{n}=c_{n}>0$ поставленный выше вопрос тривиален. Поскольку дискретный спектр в этом случае вешествен, в качестве предельного множества кроме пустого могут выступать только точки \pm 1 (каждая в отдельности или обе сразу). При этом хорошо известно [4], что уже сушествование первого момента

$$
\sum_{n} n\left(\left|a_{n}-\frac{1}{2}\right|+\left|b_{n}\right|\right)<\infty
$$

обеспечивает конечность дискретного спектра оператора $J$.

Настоящая статья возникла как попытка понять замечательные работы [1], [2], содержащие синтез идей теории операторов и теории функций в круге, которые, на наш взгляд, не получили должной оценки специалистов. Сравнительная простота дискретной модели позволила устранить ряд неточностей и упростить рассуждения работ [1], [2], применяя в $§ 2$ развитую технику определителей возмущения [5], [6]. Параграф 3 следует в основном работе Л. Карлесона [7] о множествах нулей голоморфных функций в единичном круге, результаты которой позволяют получить оценку (1.5) и завершить доказательство теоремы 1 . В $\S 4$ приводятся необходимые сведения из задачи рассеяния для вещественных матриц, которые применяются в $\S 5$ для доказательства теоремы 2. Для удобства читателей в приложении приведены некоторые результаты об определителях возмушения для комплексных якобиевых матриц.

Отметим в заключение, что теория комплексных якобиевых матриц представляет собой интенсивно развивающийся раздел теории операторов, находящий применения в теории формальных ортогональных полиномов, $J$-непрерьвных дробей и аппроксимаций Паде (см. [8]-[12]).

\section{§2. Определители возмущения и решение Йооста}

Техника определителей возмущения в спектральной теории якобиевых матриц развита в недавней работе Р. Киллипа и Б. Саймона [6; раздел 2]. Основные результаты этой работы касаются вещественных симметрических матриц $\left(b_{n}=\overline{b_{n}}, a_{n}=\right.$ $c_{n}>0$ ), однако та часть из них, о которой пойдет речь далее, ни в коей мере не использует эту специфику, и результаты сохраняются для комплексных якобиевых матриц (1.2) (см. приложение). В дальнейшем мы предполагаем сушествование первого момента

$$
\sum_{k=0}^{\infty}(k+1)\left(\left|a_{k}-\frac{1}{2}\right|+\left|b_{k}\right|+\left|c_{k}-\frac{1}{2}\right|\right)<\infty
$$

хотя некоторые результаты (в ослабленном виде) верны при менее ограничительных предположениях. 
При условии (2.1) оператор $J$ есть ядерное возмушение оператора $J_{0}$, т.е. $\Delta J=$ $J-J_{0} \in \mathscr{S}_{1}$, так что при $\lambda \notin[-1,1]$

$$
(J-\lambda)\left(J_{0}-\lambda\right)^{-1}=I+\Delta J\left(J_{0}-\lambda\right)^{-1},
$$

и тем самым при $|z|<1$ корректно определен определитель возмушения [5; гл. IV .3]:

$$
\Delta(z, J):=\operatorname{det}(J-\lambda)\left(J_{0}-\lambda\right)^{-1}, \quad \lambda(z)=\frac{z+z^{-1}}{2} .
$$

Основные свойства определителя возмущения содержатся в следующем утверждении (см., например, [5; гл. IV, §3], [6; теорема 2.5]).

УТВЕРЖДЕНИЕ 2.1. При условии (2.1) функиия $\Delta$ принадлежсит алгебре $\mathscr{A}$ голоморфных в единичном круге $\mathbb{D}=\{z:|z|<1\}$ и непрерывных вплоть до границы функций; $\Delta\left(z_{0}\right)=0$ при $\left|z_{0}\right|<1$ в том и только том случае, если $\lambda\left(z_{0}\right) \in \sigma_{d}(J)$, при этом порядок нуля равен алгебрачческой кратности собственного значения $\lambda\left(z_{0}\right)$.

Ключевую роль в теории играет аппроксимационное соотношение для определителей возмущения. Обозначим через

$$
J_{m}=\left(\begin{array}{ccccc}
b_{0} & c_{0} & & & \\
a_{0} & b_{1} & c_{1} & & \\
& \ddots & \ddots & \ddots & \\
& & \ddots & \ddots & c_{m-1} \\
& & & a_{m-1} & b_{m}
\end{array}\right), \quad J_{0, m}=\left(\begin{array}{ccccc}
0 & \frac{1}{2} & & & \\
\frac{1}{2} & 0 & \frac{1}{2} & & \\
& \ddots & \ddots & \ddots & \\
& & \ddots & \ddots & \frac{1}{2} \\
& & \frac{1}{2} & 0
\end{array}\right)
$$

матрицы порядка $m+1$, полученные из матриц $J$ и $J_{0}$ соответственно. Тогда [6; $(2.59)]$

$$
\Delta(z, J)=\lim _{m \rightarrow \infty} \operatorname{det}\left(\frac{J_{m}-\lambda}{J_{0, m}-\lambda}\right)
$$

равномерно на компактных подмножествах $\mathbb{D}$ (доказательство соотношения $(2.2)$ дано в приложении).

Наряду с исходными матрицами $J, J_{0}$ рассмотрим так называемые ассоииированные матрицы $J^{(n)}, J_{0}^{(n)}$, которые получаются вычеркиванием первых $n+1$ строк и столбцов у матриц $J, J_{0}$ соответственно:

$$
J^{(n)}=\left(\begin{array}{cccc}
b_{n+1} & c_{n+1} & & \\
a_{n+1} & b_{n+2} & c_{n+2} & \\
& \ddots & \ddots & \ddots
\end{array}\right), \quad J_{0}^{(n)}=J_{0},
$$

и $J^{(-1)}=J$. Матрицы $J_{m}^{(n)}$ определяются естественньм образом. Разлагая определитель $\operatorname{det}\left(J_{m}-\lambda\right)$ по первой строке, мы получаем после деления на $\operatorname{det}\left(J_{0, m}-\lambda\right)$

$$
\begin{aligned}
\operatorname{det}\left(\frac{J_{m}-\lambda}{J_{0, m}-\lambda}\right)= & \left(b_{0}-\lambda\right) \operatorname{det}\left(\frac{J_{m-1}^{(0)}-\lambda}{J_{0, m-1}-\lambda}\right) \operatorname{det}\left(\frac{J_{0, m-1}-\lambda}{J_{0, m}-\lambda}\right) \\
& -a_{0} c_{0} \operatorname{det}\left(\frac{J_{m-2}^{(1)}-\lambda}{J_{0, m-2}-\lambda}\right) \operatorname{det}\left(\frac{J_{0, m-2}-\lambda}{J_{0, m}-\lambda}\right) .
\end{aligned}
$$


Далее, непосредственно вычисляется (ср. [6; (2.10)])

$$
\lim _{m \rightarrow \infty} \operatorname{det}\left(\frac{J_{0, m-j}-\lambda}{J_{0, m}-\lambda}\right)=(-2 z)^{j}, \quad j \in \mathbb{N}=\{1,2, \ldots\},
$$

так что предельный переход в (2.3) при $m \rightarrow \infty$ с учетом (2.2) приводит к соотношению

$$
\Delta(z, J)=\left(\lambda-b_{0}\right)\left(2 z \Delta\left(z, J^{(0)}\right)\right)-a_{0} c_{0}\left(4 z^{2} \Delta\left(z, J^{(1)}\right)\right) .
$$

Поскольку (2.4) справедливо для любой матрипы $J$, запишем его для $J=J^{(n)}$ :

$$
\Delta\left(z, J^{(n)}\right)=\left(\lambda-b_{n+1}\right)\left(2 z \Delta\left(z, J^{(n+1)}\right)\right)-a_{n+1} c_{n+1}\left(4 z^{2} \Delta\left(z, J^{(n+2)}\right)\right) .
$$

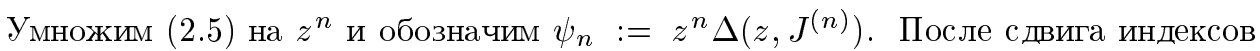
получаем

$$
\psi_{m-1}(z)+2 b_{m} \psi_{m}(z)+4 a_{m} c_{m} \psi_{m+1}(z)=2 \lambda \psi_{m}(z), \quad m \geqslant 0 .
$$

Еще одно важное свойство определителей возмущения содержится в следующем результате [6; утверждение 2.14], доказательство которого дано в приложении.

УТВЕРЖДЕНИЕ 2.2. При условии (2.1) имеет место соотношение

$$
\lim _{m \rightarrow \infty} \Delta\left(z, J^{(m)}\right)=1
$$

равномерно в замкнутом круге $\overline{\mathbb{D}}$.

Тем самым для вещественной симметрической матрицы $J$ решение $\psi_{m}(z)$ уравнения (2.6) с точностью до константы (относительно спектрального параметра) совпадает с решением Йоста уравнения $(J-\lambda) \psi=0$ (см. [13]).

При условии (2.1) обозначим

$$
H(n):=\sum_{j=n}^{\infty}\left(\left|2 b_{j}\right|+\left|4 a_{j} c_{j}-1\right|\right), \quad H(n, m):=\prod_{j=n+1}^{n+m-1}(1+H(j)) .
$$

Как легко видеть, $\{H(n)\} \in \ell^{1}$ и последовательности $H(n)$ и $H(n, m)$ монотонно убывают.

Основным результатом данного параграфа является

ТЕОРема 2.3. При условии (2.1) тейлоровские коэффициенты определителя возмущения

$$
\Delta(z, J)=\sum_{j=0}^{\infty} \delta_{j} z^{j}
$$

допускают оценку

$$
\left|\delta_{j}\right| \leqslant \prod_{j=1}^{\infty}(1+H(j)) \sum_{m=[j / 2]}^{\infty}\left(\left|2 b_{m}\right|+\left|4 a_{m} c_{m}-1\right|\right),
$$

где $[x]$ есть челая часть $x$. В частности, определитель возмущения $\Delta$ принадлежит пространству $W_{+}$абсолютно сходящихся рядов Тейлора. 
ДокАЗАТЕЛЬСТво. Покажем, что $\psi_{n}$ удовлетворяет “дискретному интегральному" уравнению вольтерровского типа. Для этого введем функцию

$$
G(n, m, z)= \begin{cases}2 \frac{z^{m-n}-z^{n-m}}{z-z^{-1}}, & m \geqslant n \\ 0, & m<n\end{cases}
$$

для которой выполняется

$$
\frac{1}{2} G(n, m+1, z)+\frac{1}{2} G(n, m-1, z)-\lambda G(n, m, z)=\delta(n, m),
$$

где $\lambda=\left(z+z^{-1}\right) / 2, \delta(n, m)$ - символ Кронекера. Умножим теперь соотношение $(2.10)$ на $\psi_{m},(2.6)$ - на $G(n, m, z) / 2$, вычтем из первого второе и просуммируем полученные равенства по $m$ от $n$ до $N$. Имеем

$$
\begin{aligned}
\psi_{n}= & \sum_{m=n}^{N}\left(-b_{m} G(n, m, z)+\left(\frac{1}{2}-2 a_{m-1} c_{m-1}\right) G(n, m-1, z)\right) \psi_{m} \\
& +\frac{1}{2} G(n, N+1, z) \psi_{N}-2 a_{N} c_{N} G(n, N, z) \psi_{N+1} .
\end{aligned}
$$

Устремим теперь $N \rightarrow \infty$, учитьвая явньй вид ядра $G$ и соотношение (2.7):

$$
\psi_{n}(z)=z^{n}+\sum_{m=n+1}^{\infty} M(n, m, z) \psi_{m}(z), \quad n=-1,0,1, \ldots,
$$

где

$$
M(n, m, z)=-b_{m} G(n, m, z)+\left(\frac{1}{2}-2 a_{m-1} c_{m-1}\right) G(n, m-1, z),
$$

причем, очевидным образом, $M(n, n, z)=0$. Удобно преобразовать $(2.11)$, введя переменные

$$
\Delta\left(z, J^{(n)}\right)=z^{-n} \psi_{n}, \quad \widehat{M}(n, m, z)=M(n, m, z) z^{m-n}
$$

так что

$$
\Delta\left(z, J^{(n)}\right)=1+\sum_{m=n+1}^{\infty} \widehat{M}(n, m, z) \Delta\left(z, J^{(m)}\right)
$$

или иначе

$$
\Delta\left(z, J^{(n)}\right)-1=\sum_{m=n+1}^{\infty} \widehat{M}(n, m, z)+\sum_{m=n+1}^{\infty} \widehat{M}(n, m, z)\left(\Delta\left(z, J^{(m)}\right)-1\right) .
$$

Ясно, что $\widehat{M}$ - полином по переменной $z$, и так как при $|z| \leqslant 1$

$$
\begin{gathered}
\left|G(n, m, z) z^{m-n}\right|=2\left|z^{2(m-n)}-1\right|\left|z-z^{-1}\right|^{-1} \leqslant 2|z||m-n|, \\
\left|G(n, m-1, z) z^{m-n}\right| \leqslant 2|z|^{2}|m-n-1|<2|z||m-n|
\end{gathered}
$$


TO

$$
|\widehat{M}(n, m, z)| \leqslant|z||m-n|\left(\left|2 b_{m}\right|+\left|1-4 a_{m-1} c_{m-1}\right|\right), \quad|z| \leqslant 1 .
$$

При условии (2.1) ряд

$$
g(n, z)=\sum_{m=n+1}^{\infty} \widehat{M}(n, m, z)
$$

сходится абсолютно и равномерно в замкнутом единичном круге $\overline{\mathbb{D}}$ и в силу $(2.13)$

$$
|g(n, z)| \leqslant \sum_{m=n+1}^{\infty} m h_{m}, \quad h_{m}=\left|2 b_{m}\right|+\left|1-4 a_{m-1} c_{m-1}\right| .
$$

Стандартный метод последовательных приближений (ср. [14; лемма 7.8]) дает

$$
\left|\Delta\left(z, J^{(n)}\right)-1\right| \leqslant \exp \left(\sum_{m=1}^{\infty} m h_{m}\right) \sum_{m=n+1}^{\infty} m h_{m}, \quad n \geqslant-1, \quad|z| \leqslant 1 .
$$

Разложим функцию $\Delta\left(z, J^{(n)}\right)$ в ряд Тейлора

$$
\Delta\left(z, J^{(n)}\right)=1+\sum_{j=1}^{\infty} \varkappa(n, j) z^{j}
$$

и оценим ее тейлоровские коэффициенты. По неравенству Коши

$$
|\varkappa(n, j)| \leqslant C \sum_{m=n+1}^{\infty} m h_{m}
$$

где через $C$ мы будем обозначать положительные постоянные, не зависящие от пространственных переменных и спектрального параметра. В частности, $\varkappa(n, j) \rightarrow 0$ при $n \rightarrow \infty$ и фиксированном $j$.

Более точная оценка учитывает зависимость от второго индекса. Подставим разложение (2.14) в (2.5) и приравняем соответствуюшие коэффициенты:

$\varkappa(n, j+1)=\varkappa(n+1, j-1)-\sum_{m=n+1}^{\infty}\left(2 b_{m} \varkappa(m, j)+\left(4 a_{m} c_{m}-1\right) \varkappa(m+1, j-1)\right)$

при $j \geqslant 2$,

$$
\varkappa(n, 1)=-2 \sum_{m=n+1}^{\infty} b_{m}, \quad \varkappa(n, 2)=-\sum_{m=n+1}^{\infty}\left(2 b_{m} \varkappa(m, 1)+\left(4 a_{m} c_{m}-1\right)\right) .
$$

Индукция по $j$ с учетом (2.15) приводит к оценке

$$
|\varkappa(n, j)| \leqslant H(n, j) H\left(n+1+\left[\frac{j}{2}\right]\right), \quad n \geqslant-1,
$$

где $H(n), H(n, m)$ определены в (2.8). Поэтому

$$
|\varkappa(n, j)| \leqslant \prod_{j=1}^{\infty}(1+H(j)) \sum_{m=n+1+[j / 2]}^{\infty}\left(\left|2 b_{m}\right|+\left|4 a_{m} c_{m}-1\right|\right) .
$$

Искомая оценка (2.9) есть (2.16) с $n=-1$. Теорема доказана. 
СлЕДСТВИЕ 2.4. Пусть для матрицы $J(1.2)$ конечен момент порядка $n+1$

$$
M_{n+1}:=\sum_{k=0}^{\infty}(k+1)^{n+1}\left(\left|a_{k}-\frac{1}{2}\right|+\left|b_{k}\right|+\left|c_{k}-\frac{1}{2}\right|\right)<\infty .
$$

Тогда п-я производная $\Delta^{(n)}(z)$ определителя возмущения принадлежит $W_{+} u$

$$
\max _{z \in \overline{\mathbb{D}}}\left|\Delta^{(n)}(z)\right| \leqslant C(J) \frac{4^{n}}{n+1} M_{n+1},
$$

где положительная константа $C(J)$ зависит лишь от $J$.

ДокАЗАТЕЛЬСтво. Утверждение очевидным образом вытекает из условия (2.17), представления

$$
\Delta^{(n)}(z)=\sum_{j=0}^{\infty}(j+1) \cdots(j+n) \delta_{j+n} z^{j}
$$

и оценок $(2.9)$.

\section{§3. Множества нулей для классов голоморфных в единичном круге функций}

Пусть $X$ - некоторый класс функций из алгебры $\mathscr{A}$. Замкнутое множество $E$ на единичной окружности $\mathbb{T}$ называется множеством единственности для $X$, если не существует нетривиальной функции $f \in X$, равной нулю на $E$. В противном случае говорят о множестве нулей для $X$. Если $X=\mathscr{A}$, то согласно тереме Фату [15] множество $E$ есть множество нулей для $\mathscr{A}$ в том и только том случае, если $E$ имеет лебегову меру нуль: $|E|=0$. Если $X$ есть подкласс $\mathscr{A}$, то множества нулей, вообще говоря, обладают дополнительными свойствами. Исследование (метрических) свойств множеств нулей восходит к А. Берлингу [16] и Л. Карлесону [7] (см. также [17]). Например, обозначим через $\mathscr{A}_{\infty}$ множество всех функций из $\mathscr{A}$, все производные которых также принадлежат $\mathscr{A}$. Замкнутое множество $E=\mathbb{T} \backslash \bigcup_{j} l_{j}$ со смежными дугами $l_{j}$ есть множество нулей для $\mathscr{A}_{\infty}$ в том и только том случае, если

$$
\sum_{j}\left|l_{j}\right| \ln \frac{1}{\left|l_{j}\right|}<\infty \Leftrightarrow \int_{\mathbb{T}}|\ln \rho(\zeta, E)| d m<\infty, \quad \rho(\zeta, E)=\operatorname{dist}(\zeta, E),
$$

где $d m$ - нормированная мера Лебега на $\mathbb{T}$. Нас будут интересовать некоторые специальные подклассы класса $\mathscr{A}_{\infty}$, и для множеств нулей возникнет условие более сильное, чем (3.1).

Пусть $g \in \mathscr{A}_{\infty}$ и

$$
G_{n}(g)=G_{n}:=\max _{z \in \overline{\mathbb{D}}}\left|g^{(n)}(z)\right|, \quad n \in \mathbb{Z}_{+}
$$


Следуюший результат (формула Тейлора с остаточным членом) легко получается индукцией по $n$.

Лемма 3.1. Пусть $g \in \mathscr{A}_{\infty}$, тогда для любого $n \in \mathbb{N}$ и любих точек $z, w$ из $\overline{\mathbb{D}}$ справедливо неравенство

$$
\left|g(z)-\sum_{k=0}^{n-1} \frac{g^{(k)}(w)}{k !}(z-w)^{k}\right| \leqslant \frac{G_{n}}{n !}|z-w|^{n} .
$$

Введем нулевое множество функции $g$ на $\mathbb{T}$ :

$$
F(g)=F:=\left\{\zeta \in \mathbb{T}: g^{(n)}(\zeta)=0 \text { при всех } n \in \mathbb{Z}_{+}\right\} .
$$

Тогда $F$ есть замкнутое множество меры нуль, которое мы отождествим с множеством точек на $[0,2 \pi)$. Обозначим через $F_{t}$ замкнутое множество точек, расстояние от которых до $F$ не превосходит $t$, и положим $\varphi_{F}(t)=\varphi(t)=\left|F_{t}\right|$. Свойства функции $\varphi$ при $t \rightarrow 0$ играют решаюшую роль при изучении множеств нулей для классов функций в $\mathscr{A}_{\infty}$, допускающих оценки на производные (3.2). Функция $\varphi$ известна также как функция распределения функции $d_{F}(x)=\operatorname{dist}(x, F), x \in \mathbb{R}$. По формуле замены переменной для любой измеримой функции $h$

$$
\int_{F_{s}} h\left(d_{F}(t)\right) d t=\int_{0}^{s} h(u) d \varphi(u) .
$$

Для функции $g \in \mathscr{A}_{\infty}$ введем величину (ср. [1])

$$
T(s):=\inf _{k \geqslant 0} \frac{G_{k}(g)}{k !} s^{k}, \quad s>0 .
$$

Лемма 3.2. Пусть $g \in \mathscr{A}_{\infty}$ u $g \neq 0$. Тогда

$$
\int_{0}^{2 \pi} \ln T(s) d \varphi(s)>-\infty .
$$

ДокаЗАТЕЛЬСтво. Положим в (3.3) $w=\exp \left(i \theta_{0}\right), \theta_{0} \in F$, и $z=\exp (i \theta)$. Тогда

$$
\left|g\left(e^{i \theta}\right)\right| \leqslant \frac{G_{n}}{n !}\left|\theta-\theta_{0}\right|^{n} .
$$

Правая часть этого неравенства содержит два параметра ( $n$ и $\left.\theta_{0}\right)$, по которым можно ее минимизировать:

$$
\left|g\left(e^{i \theta}\right)\right| \leqslant \inf _{n \geqslant 0} \frac{G_{n}}{n !}\left(d_{F}(\theta)\right)^{n}=T\left(d_{F}(\theta)\right), \quad \ln \left|g\left(e^{i \theta}\right)\right| \leqslant \ln T\left(d_{F}(\theta)\right) .
$$

Остается учесть (3.4) и известное свойство

$$
\int_{0}^{2 \pi} \ln \left|g\left(e^{i \theta}\right)\right| d \theta>-\infty
$$

Лемма доказана.

Введем, наконец, основное для нас множество, связанное с нулями функции $g$ внутри круга:

$$
E(g)=E:=\left\{\zeta \in \mathbb{T}: \exists\left\{z_{n}\right\} \in \mathbb{D}, z_{n} \rightarrow \zeta, g\left(z_{n}\right)=0\right\} .
$$


Лемма 3.3. Имеем $E \subset F$.

ДокАЗАТЕльСтво. Предположим, что $g^{(k)}(w)=0, k=0,1, \ldots, n-2$, и $g^{(n-1)}(w) \neq 0$ для некоторого $w \in E$. В силу $(3.3)$

$$
\left|g(z)-\frac{g^{(n-1)}(w)}{(n-1) !}(z-w)^{n-1}\right| \leqslant \frac{G_{n}}{n !}|z-w|^{n} .
$$

Остается положить $z=z_{k}$ и устремить $k \rightarrow \infty$. Противоречие доказьвает лемму.

ДоКАЗАТЕЛЬСтво теОРЕмЫ 1 опирается на следствие 2.4. Для матриц $J \in$ $\mathscr{P}(\beta)(1.4)$ условие (2.17) заведомо выполняется при всех $n$, и моменты $M_{r}$ легко оцениваются. Поскольку

$$
\left|a_{k}-\frac{1}{2}\right|+\left|b_{k}\right|+\left|c_{k}-\frac{1}{2}\right| \leqslant C \exp \left(-C(k+1)^{\beta}\right), \quad 0<\beta<1
$$

(через $C$ мы обозначаем положительные константы, зависящие лишь от исходной матрицы $J)$, мы имеем

$$
M_{r} \leqslant C \sum_{k=0}^{\infty}(k+1)^{r} \exp \left(-\frac{C}{2}(k+1)^{\beta}\right) \exp \left(-\frac{C}{2}(k+1)^{\beta}\right) .
$$

Элементарный анализ функции $u(x)=x^{r} \exp \left(-C x^{\beta} / 2\right)$ дает

$$
\max _{x \geqslant 0} u(x)=u\left(x_{0}\right)=\left(\frac{2 r}{C \beta}\right)^{r / \beta} e^{-r / \beta}=\left(\frac{2}{C \beta e}\right)^{r / \beta} r^{r / \beta}, \quad x_{0}=\left(\frac{2 r}{C \beta}\right)^{1 / \beta},
$$

так что

$$
M_{r} \leqslant B\left(\frac{2}{C \beta e}\right)^{r / \beta} r^{r / \beta}, \quad B=C \sum_{k=0}^{\infty} \exp \left(-\frac{C}{2}(k+1)^{\beta}\right) .
$$

Таким образом,

$$
G_{n}(\Delta)=\max _{z \in \overline{\mathbb{D}}}\left|\Delta^{(n)}(z)\right| \leqslant C \frac{4^{n}}{n+1}\left(\frac{2}{C \beta e}\right)^{(n+1) / \beta}(n+1)^{(n+1) / \beta}, \quad n \geqslant 0 .
$$

Неравенства

$$
\left(\frac{n+1}{n}\right)^{n / \beta}<e^{1 / \beta}, \quad(n+1)^{1 / \beta}<e^{(n+1) / \beta}, \quad(n+1)^{(n+1) / \beta}<e^{1 / \beta} n^{n / \beta} e^{(n+1) / \beta}
$$

приводят к оценке

$$
G_{n}(\Delta) \leqslant C C_{1}^{n} n^{n / \beta}, \quad n \geqslant 0 .
$$

Иными словами, определитель возмушения для матриц $J \in \mathscr{P}(\beta)$ принадлежит классу Жевре $\mathscr{G}_{\beta}$. 
Перейдем теперь к оценке функции $T(3.5)$. Так как $n !>n^{n} e^{-n}$, то

$$
T(s) \leqslant C \inf _{n}\left(C_{1} e s\right)^{n} n^{n / \beta-n}=C \inf _{n} t^{n} n^{\alpha n}
$$

где $t=C_{1}$ es $<1 / 2$ при достаточно малых $s, \alpha=\beta^{-1}-1>0$. Элементарный анализ функции $v(x)=t^{x} x^{\alpha x}$ показьвает, что

$$
\min _{x \geqslant 0} v(x)=v\left(x_{1}\right)=\exp \left(-\frac{\alpha}{e} t^{-1 / \alpha}\right), \quad x_{1}=\frac{1}{e} t^{-1 / \alpha} \gg 1 .
$$

Хотя число $x_{1}$, вообще говоря, не целое, полагая $n=\left[x_{1}\right]$, нетрудно убедиться, что

$$
T(s) \leqslant C \exp \left(-C\left(\frac{1}{s}\right)^{\beta /(1-\beta)}\right), \quad s \leqslant s_{0}
$$

Применяя лемму 3.2 , мы заключаем, что

$$
\int_{0}^{a} \frac{d \varphi_{F}(s)}{s^{\beta /(1-\beta)}}<\infty, \quad 0<a<1, \quad F=F(\Delta) .
$$

Предположим, что $0<\beta<\frac{1}{2}$. Как показано в [7], сходимость интеграла (3.7) эквивалентна сходимости ряда

$$
\sum_{j=1}^{\infty}\left|l_{j}\right|^{(1-2 \beta) /(1-\beta)}<\infty,
$$

где $\left\{l_{j}\right\}$ - смежные интервалы замкнутого множества $F$ (как множества на $[0,2 \pi]$ ), и тем самым

$$
\tau(F) \leqslant \frac{1-2 \beta}{1-\beta} .
$$

Неравенство $\operatorname{dim} F \leqslant \tau(F)$ есть общий факт теории фрактальных размерностей (см. [18]). Но по лемме $3.3 E=E(\Delta) \subset F$, так что в силу монотонности размерностей

$$
\operatorname{dim} E \leqslant \tau(E) \leqslant \frac{1-2 \beta}{1-\beta} .
$$

Неравенство (1.5) вытекает теперь из очевидной связи меж ду $E_{J}$ и $E$.

Рассмотрим случай $\beta=1 / 2$, так что

$$
\int_{0}^{a} \frac{d \varphi_{F}(s)}{s}<\infty
$$

Покажем, что последнее невозможно для непустого $F$. Предположим, что смежные дуги $l_{j}=\left(\alpha_{j}, \beta_{j}\right)$ занумерованы в порядке убывания длины, т.е. $\left|l_{1}\right| \geqslant\left|l_{2}\right| \geqslant \cdots$. Пусть

$$
0<t_{1}<t_{2}<\frac{1}{2}\left|l_{1}\right|=\frac{\beta_{1}-\alpha_{1}}{2} .
$$


По определению функции $\varphi$

$$
\varphi\left(t_{2}\right)-\varphi\left(t_{1}\right)=\left|\left\{x: t_{1}<\operatorname{dist}(x, F) \leqslant t_{2}\right\}\right| .
$$

Для интервала $I\left(t_{1}, t_{2}\right)=\left(\alpha_{1}+t_{1}, \alpha_{1}+t_{2}\right)$, очевидно, выполняется

$$
I\left(t_{1}, t_{2}\right) \subset\left\{x: t_{1}<\operatorname{dist}(x, F) \leqslant t_{2}\right\},
$$

так что $\varphi\left(t_{2}\right)-\varphi\left(t_{1}\right) \geqslant t_{2}-t_{1}$. Поэтому мера $d \varphi_{F}$ мажорирует меру Лебега, и интеграл (3.8) расходится. Тем самым мы приходим к выводу: если $J \in \mathscr{P}(1 / 2)$, то множество $F$ (а значит, и $E$ ) пусто, что означает конечность дискретного спектра $\sigma_{d}(J)$. Теорема доказана.

ЗАмечАниЕ 1. Следствие 2.4 позволяет получить условия на "массивность" множества $E_{J}$ в гораздо более общих ситуациях. Так если для матрицы $J$ выполнено (2.17), то $\Delta \in \mathscr{A}_{\infty}$ и, значит, для множества $E(\Delta)$ справедливо (3.1) и то же условие (после очевидной переформулировки для отрезка $[-2,2])$ верно для $E_{J}$. Другим примером может служить класс якобиевых матриц $J$ с условием

$$
\left|a_{k}-\frac{1}{2}\right|+\left|b_{k}\right|+\left|c_{k}-\frac{1}{2}\right| \leqslant C \exp \left(-C(\ln (k+1))^{\gamma}\right), \quad \gamma>1,
$$

содержащий класс $\mathscr{P}(\beta)$. Нетрудно убедиться, что определитель возмушения таких матриц удовлетворяет оценке

$$
\max _{z \in \overline{\mathbb{D}}}\left|\Delta^{(n)}(z)\right| \leqslant C \exp \left(C n^{p}\right), \quad p=\frac{\gamma}{\gamma-1}>1 .
$$

Функции с такими оценками на производные изучены Б. Тейлором и Д. Вильямсом в [19], где получено условие на нулевое множество

$$
\int_{\mathbb{T}}|\ln \rho(\zeta, E)|^{q} d m<\infty, \quad \frac{1}{q}+\frac{1}{p}=1 .
$$

С другой стороны, конечность дискретного спектра матриц $J \in \mathscr{P}(1 / 2)$ обусловлена квазианалитичностью класса Жевре $\mathscr{G}_{1 / 2}$, т.е. отсутствием нетривиальной функции $f$ такой, что $f^{(n)}\left(\zeta_{0}\right)=0$ при всех $n \geqslant 0$ и некотором $\zeta_{0} \in \mathbb{T}$. Известньй критерий квазианалитичности [20] позволяет получить несколько более сильное, чем (1.4) с $\beta=1 / 2$, условие конечности дискретного спектра.

ЗАмечание 2. Определитель возмушения матрицы $J \in \mathscr{P}(1 / 2)$ может иметь граничные нули на единичной окружности, что видно уже на простом примере

$$
J: \quad a_{n}=c_{n}=\frac{1}{2}, \quad b_{0}=e^{i \theta}, \quad b_{1}=b_{2}=\cdots=0,
$$

для которого легко вычисляется $\Delta(z, J)=1-e^{i \theta} z$. Эти точки (точнее, их образы при отображении Жуковского) известны как спектральные особенности. В указанном примере при $\theta \neq 0, \pi$ матрица $J$ имеет пустой дискретный спектр и одну спектральную особенность в точке $\cos \theta \in(-1,1)$. При общем условии $(2.1)$ спектральные особенности образуют замкнутое множество меры нуль. Ясно, что матрица $J \in \mathscr{P}(1 / 2)$ может иметь не более конечного числа спектральных особенностей (ср. [3]). В самом деле, в противном случае сушествовала бы точка $\zeta_{0} \in \mathbb{T}$, для которой $\Delta^{(n)}\left(\zeta_{0}, J\right)=0$ при всех $n \geqslant 0$ (cp. (3.3)), что противоречит квазианалитичности класса $\mathscr{G}_{1 / 2}$. 


\section{$\S 4$. О данных рассеяния вещественной симметрической матрицы Якоби}

В этом параграфе, основываясь на некоторых фактах задачи рассеяния для полубесконечной вещественной матрищы Якоби [4], [13], [21], мы изучаем связь между коэффициентами Фурье функции рассеяния и скоростью стабилизации элементов якобиевой матрицы в отсутствие дискретного спектра и резонансов. Основной результат - лемма 4.2 .

Пусть

$$
J=\left(\begin{array}{ccccc}
b_{1} & a_{1} & & & \\
a_{1} & b_{2} & a_{2} & & \\
& \ddots & \ddots & \ddots & \\
& & \ddots & \ddots & \ddots
\end{array}\right)
$$

- матрица Якоби с элементами $a_{n}>0, b_{n}=\overline{b_{n}}$, удовлетворяющими условию (1.5). Положим $a_{0}=1$. Как известно (см. [4], [13], [21]), у уравнения

$$
a_{n-1} y_{n-1}+b_{n} y_{n}+a_{n} y_{n+1}=\frac{1}{2}\left(z+z^{-1}\right) y_{n}, \quad z \in \overline{\mathbb{D}}, \quad n \geqslant 1,
$$

есть решение $f_{n}(z)$, называемое решением Йоста, которое обладает следующими свойствами:

i) $\lim _{n \rightarrow \infty} f_{n}(z) z^{-n}=1$ равномерно по $z \in \overline{\mathbb{D}}$;

ii) при всех $n \geqslant 0$ функции $f_{n}(z)$ принадлежат алгебре $W_{+}$абсолютно сходящихся рядов Тейлора, при этом $f_{n}(\bar{\zeta})=\overline{f_{n}(\zeta)}, \zeta \in \mathbb{T}$;

iii) если функция $f_{0}(z)$, называемая функиией Йоста, имеет нули в $\overline{\mathbb{D}} \backslash\{ \pm 1\}$, то они вещественные, простые, их конечное число и их образы в $\lambda$-плоскости являются точками дискретного спектра оператора $J$;

iv) решения $f_{n}(\zeta)$ и $\overline{f_{n}(\zeta)}$ линейно независимы при $\zeta \in \mathbb{T} \backslash\{ \pm 1\}$ и

$$
\langle f, \bar{f}\rangle=\frac{\zeta^{-1}-\zeta}{2},
$$

где $\langle f, \bar{f}\rangle:=a_{n-1}\left(f_{n-1} \overline{f_{n}}-f_{n} \overline{f_{n-1}}\right)$ - вронскиан этих решений.

В силу свойств і) и іi) имеет место представление

$$
f_{n}(z)=\sum_{m=n}^{\infty} K(n, m) z^{m}, \quad z \in \overline{\mathbb{D}}, \quad n \in \mathbb{Z}_{+},
$$

где элементы $K(n, m)$ матрищы, называемой оператором преобразования, вещественны и связаны с элементами матрицы $J$ соотношениями [14], [22]

$$
\begin{aligned}
& a_{n}=\frac{K(n+1, n+1)}{2 K(n, n)}, \text { т.е. } K(n, n)=\prod_{j=n}^{\infty}\left(2 a_{j}\right)^{-1}, \\
& b_{n}=\frac{K(n, n+1)}{2 K(n, n)}-\frac{K(n-1, n)}{2 K(n-1, n-1)}, \quad n \geqslant 1 .
\end{aligned}
$$


Отметим, что функция Йоста связана (с точностью до множителя, не зависящего от спектральной переменной) с определителем возмушения матрицы $J$ формулой $[6 ;(2.64)]$

$$
f_{0}(z)=\left(\prod_{j=1}^{\infty} 2 a_{j}\right)^{-1} \Delta(z, J)
$$

Пусть $S(\zeta)=\overline{f_{0}(\zeta)}\left(f_{0}(\zeta)\right)^{-1}-$ функция рассеяния матрицы $J, \zeta \in \mathbb{T}$. Так как $f_{0}(\zeta) \neq 0, \zeta \in \mathbb{T}$ (в силу предположения об отсутствии резонанса $f_{0}( \pm 1) \neq 0$ ), то $S(\zeta)$ - непрерывная функция на $\mathbb{T}$, причем $\overline{S(\zeta)}=S(\bar{\zeta})=S^{-1}(\zeta)$. Положим

$$
F(n):=-\frac{1}{2 \pi} \int_{-\pi}^{\pi} S\left(e^{i \theta}\right) e^{i n \theta} d \theta
$$

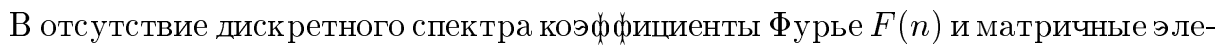
менты $K(n, m)$ оператора преобразования связаны уравнением обратной задачи рассеяния (уравнением Марченко, см. [4], [13])

$$
\frac{\delta(n, m)}{K(n, n)}=K(n, m)+\sum_{l=n}^{\infty} K(n, l) F(l+m), \quad n, m \in \mathbb{Z}_{+} .
$$

Известно [14; (10.87)], что при условии (1.5) имеет место неравенство

$$
\sum_{n=1}^{\infty} n|F(n+2)-F(n)|<\infty
$$

Введем дискретный аналог оператора Гельфанда-Левитана

$$
\mathscr{F}_{n}: \ell^{1}\left(\mathbb{Z}_{+}\right) \rightarrow \ell^{1}\left(\mathbb{Z}_{+}\right), \quad\left(\mathscr{F}_{n} y\right)_{j}=\sum_{m=0}^{\infty} F(2 n+m+j) y_{m}, \quad j \in \mathbb{Z}_{+} .
$$

Здесь $n \geqslant 1-$ фиксированный параметр.

ЛЕмма 4.1. При условии (4.9) оператор $\mathscr{I}+\mathscr{F}_{n}$ обратим в пространстве $\ell^{1}\left(\mathbb{Z}_{+}\right)$при каждом $n \geqslant 1$ и

$$
\left\|\left(\mathscr{I}+\mathscr{F}_{n}\right)^{-1}\right\| \rightarrow 1 \text { npu } n \rightarrow \infty .
$$

ДокАЗАТЕЛЬСТво. Положим

$$
\widehat{F}(n):=\sum_{j=0}^{\infty}|F(n+j)-F(n+j+2)|=\sum_{k=n}^{\infty}|F(k)-F(k+2)| .
$$

Поскольку $F(n) \rightarrow 0$ при $n \rightarrow \infty$, то

$$
|F(k)| \leqslant \sum_{j=0}^{\infty}|F(k+2 j)-F(k+2 j+2)|
$$


так что

$$
|F(k)|+|F(k+1)| \leqslant \widehat{F}(k) .
$$

Соответственно $\{F(k)\} \in \ell^{1}\left(\mathbb{Z}_{+}\right)$, т.е. оператор $\mathscr{F}_{n}$ является вполне непрерьвным. По альтернативе $\Phi$ редгольма оператор $\mathscr{I}+\mathscr{F}_{n}$ обратим, если уравнение

$$
\left(\mathscr{I}+\mathscr{F}_{n}\right) g=0
$$

имеет лишь тривиальное решение. Поскольку $S\left(e^{-i \theta}\right)=\overline{S\left(e^{i \theta}\right)}$, то $F(n) \in \mathbb{R}$, так что достаточно ограничиться вещественньми решениями уравнения (4.12). Пусть $g=\{g(k)\} \in \ell^{1}-$ такое решение. Введем функцию

$$
\widetilde{g}(z):=\sum_{k=0}^{\infty} g(k) z^{k}
$$

Тогда

$$
\begin{gathered}
(g, g)=\sum_{0}^{\infty}|g(k)|^{2}=\frac{1}{2 \pi} \int_{-\pi}^{\pi}\left|\widetilde{g}\left(e^{i \theta}\right)\right|^{2} d \theta \\
\left(\mathscr{F}_{n} g, g\right)=-\frac{1}{2 \pi} \int_{-\pi}^{\pi} S\left(e^{i \theta}\right) e^{2 i n \theta} \widetilde{g}^{2}\left(e^{i \theta}\right) d \theta
\end{gathered}
$$

так что

$$
0=\left(\left(\mathscr{I}+\mathscr{F}_{n}\right) g, g\right)=\frac{1}{2 \pi} \int_{-\pi}^{\pi}\left(1-\Phi\left(e^{i \theta}\right)\right)\left|\widetilde{g}\left(e^{i \theta}\right)\right|^{2} d \theta
$$

где

$$
\Phi\left(e^{i \theta}\right)=S\left(e^{i \theta}\right) e^{2 i n \theta} \widetilde{g}^{2}\left(e^{i \theta}\right)\left|\widetilde{g}\left(e^{i \theta}\right)\right|^{-2} .
$$

Предположим, что $\widetilde{g} \neq 0$. Так как $\left|\Phi\left(e^{i \theta}\right)\right|=1$, то из (4.13) следует $\Phi\left(e^{i \theta}\right) \equiv 1$, т.е. с учетом определения функции рассеяния

$$
\frac{\widetilde{g}^{2}\left(e^{i \theta}\right)}{f_{0}^{2}\left(e^{i \theta}\right)} e^{2 i n \theta}=\frac{\left|\widetilde{g}\left(e^{i \theta}\right)\right|^{2}}{\left|f_{0}\left(e^{i \theta}\right)\right|^{2}}
$$

Тем самым, для функции $h_{n}=\widetilde{g}^{2} f_{0}^{-2} e^{2 i n \theta}$ имеем $h_{n}\left(e^{i \theta}\right)=\left|h_{n}\left(e^{i \theta}\right)\right|$. Ввиду отсутствия дискретного спектра и резонансов $f_{0}(z) \neq 0$ при $z \in \overline{\mathbb{D}}$ и, следовательно, $h_{n} \in W_{+}$, так что по принципу симметрии и теореме единственности $h_{n} \equiv$ const в $\overline{\mathbb{D}}$. Но при $n \geqslant 1 h_{n}(0)=0$, т.е. $\widetilde{g} \equiv 0$. Таким образом, уравнение (4.12) имеет лишш тривиальное решение, и оператор $\mathscr{I}+\mathscr{F}_{n}$ обратим при $n \geqslant 1$. Второе утверждение леммы вытекает из неравенств

$$
\left\|\mathscr{F}_{n}\right\| \leqslant \sum_{j=0}^{\infty}|F(2 n+j)| \rightarrow 0, \quad n \rightarrow \infty
$$

и

$$
\left\|\left(\mathscr{I}+\mathscr{F}_{n}\right)^{-1}-\mathscr{I}\right\| \leqslant \frac{\left\|\mathscr{F}_{n}\right\|}{1-\left\|\mathscr{F}_{n}\right\|} .
$$


Лемма доказана.

Положим

$$
\tau(n, j)=K(n, n+j) K(n, n)-\delta(n, n+j) .
$$

Тогда уравнение (4.8) приобретает вид

$$
\tau(n, j)+\sum_{m=0}^{\infty} F(2 n+m+j) \tau(n, m)=-F(2 n+j), \quad j \geqslant 0
$$

откуда

$$
\{\tau(n, j)\}_{j \geqslant 0}=-\left(\mathscr{I}+\mathscr{F}_{n}\right)^{-1}\{F(2 n+j)\}_{j \geqslant 0} .
$$

Имеет место следуюшее утверждение, являюшееся некоторой модификацией формулы (10.110) из [14].

ЛЕмма 4.2. При условии (4.9) справедлива оценка

$$
\left|2 a_{n}-1\right|+\left|b_{n}\right| \leqslant C\left(|F(2 n-1)-F(2 n+1)|+|F(2 n)-F(2 n+2)|+\widehat{F}^{2}(2 n-2)\right),
$$

где $\widehat{F}(n)$ определено в (4.10).

ДокАЗАТЕЛЬСтво. В пространстве $\ell^{1}\left(\mathbb{Z}_{+}\right)$рассмотрим уравнение

$$
\left(\mathscr{I}+\mathscr{F}_{n}\right) x=y,
$$

или

$$
x(j)+\sum_{m=0}^{\infty} F(2 n+j+m) x(m)=y(j), \quad x=\{x(j)\}, \quad y=\{y(j)\}, \quad j \geqslant 0 .
$$

В силу обратимости оператора $\mathscr{I}+\mathscr{F}_{n}$ при $n \geqslant 1$ для решения (4.18) справедливо

$$
\|x\|_{1} \leqslant K\|y\|_{1}, \quad K=\sup _{n \geqslant 1}\left\|\left(\mathscr{I}+\mathscr{F}_{n}\right)^{-1}\right\|<\infty .
$$

Подставляя (4.19) в (4.18), получаем оценки для координат

$$
|x(j)| \leqslant \sup _{k \geqslant 2 n+j}|F(k)|\|x\|_{1}+|y(j)| \leqslant K \sup _{k \geqslant 2 n+j}|F(k)|\|y\|_{1}+|y(j)| .
$$

С учетом (4.11) и невозрастания последовательности $\{\widehat{F}(n)\}$, приходим к неравенству

$$
|x(j)| \leqslant K \widehat{F}(2 n+j)\|y\|_{1}+|y(j)| .
$$

Применим оценку (4.20) к исходному уравнению (4.15), где

$$
x(j)=\tau(n, j), \quad y(j)=-F(2 n+j) .
$$


Поскольку в силу (4.9)-(4.14)

$$
\left\|y_{n}\right\|_{1} \leqslant \sum_{k=0}^{\infty} \widehat{F}(k)=C<\infty, \quad\left|y_{n}(j)\right| \leqslant \widehat{F}(2 n+j),
$$

то (4.20) примет вид

$$
|\tau(n, j)| \leqslant C \widehat{F}(2 n+j), \quad j \geqslant 0 .
$$

Далее, запишем (4.15) с индексами $n$ и $n+1$ и вычтем второе из первого:

$$
\left(\mathscr{I}+\mathscr{F}_{n}\right)\left(\tau_{n}-\tau_{n+1}\right)=y_{n}, \quad \tau_{n}=\{\tau(n, j)\}_{j \geqslant 0}, \quad y_{n}=\left\{y_{n}(j)\right\}_{j \geqslant 0},
$$

где

$y_{n}(j)=F(2 n+2+j)-F(2 n+j)+\sum_{m=0}^{\infty}(F(2 n+j+m)-F(2 n+2+j+m)) \tau(n+1, m)$.

С учетом (4.21) непосредственно проверяется

$$
\left|y_{n}(j)\right| \leqslant|F(2 n+j)-F(2 n+2+j)|+C \widehat{F}(2 n) \widehat{F}(2 n+j), \quad\left\|y_{n}\right\| \leqslant C \widehat{F}(2 n),
$$

так что из (4.20) следует

$|\tau(n, j)-\tau(n+1, j)| \leqslant|F(2 n+j)-F(2 n+2+j)|+C \widehat{F}(2 n) \widehat{F}(2 n+1), \quad j \geqslant 0$.

Искомая оценка (4.17) вытекает из равенств (4.5), (4.6) и определения (4.14). Действительно, по (4.5)

$$
0<C_{1} \leqslant K^{2}(n, n) \leqslant C_{2}<\infty, \quad K(n, n) \rightarrow 1, \quad n \rightarrow \infty,
$$

так что в силу $(4.22)$ с $j=0$

$$
\begin{aligned}
\left|2 a_{n}-1\right| & =\frac{|K(n+1, n+1)-K(n, n)|}{K(n, n)} \leqslant C|\tau(n, 0)-\tau(n+1,0)| \\
& \leqslant C\left(|F(2 n)-F(2 n+2)|+\widehat{F}^{2}(2 n)\right) .
\end{aligned}
$$

Далее, так как $|\tau(n, 1)| \leqslant C$ (см. (4.21)), то из (4.6)

$$
\left|2 b_{n}\right|=\frac{\left|4 a_{n-1}^{2} \tau(n-1,1)-\tau(n, 1)\right|}{K^{2}(n, n)} \leqslant C\left(|\tau(n-1,1)-\tau(n, 1)|+\left|4 a_{n-1}^{2}-1\right|\right),
$$

что с учетом $(4.22)$ при $j=1,(4.23)$ и монотонности $\widehat{F}(n)$ дает

$$
\left|b_{n}\right| \leqslant C(|F(2 n-1)-F(2 n+1)|+|F(2 n)-F(2 n+2)|+\widehat{F}(2 n-2) \widehat{F}(2 n-1)) .
$$

Объединяя оценки (4.23) и (4.24) мы приходим к (4.17), что и завершает доказательство леммы 4.2 . 


\section{§5. Пример Павлова}

В основе доказательства теоремы 2 лежит “осцилляционный интеграл”, введенньй Павловым в $[2 ; \S 3]$

$$
V(z)=\int_{0}^{z} \exp (-\chi(\xi)) \cos (\gamma \chi(\xi)) d \xi
$$

где $\chi(\xi)=\left(1+\xi^{2}\right)^{\gamma-1} / 32$ и $\gamma-$ малый параметр, $0<\gamma<1$. Этот интеграл обладает следующими свойствами:

i) $V \in \mathscr{A}_{\infty}$ и голоморфна в каждой точке $\zeta \in \mathbb{T} \backslash\{ \pm i\}$;

ii) $V(0)=0$ и $|V(z)| \leqslant 1$ при $z \in \overline{\mathbb{D}}$, причем $V(z) \neq 0$ при $z \neq 0$;

iii) $\operatorname{Im} V(z) \operatorname{Im} z>0$ при $z \notin \mathbb{R}$;

iv) сушествует последовательность точек на мнимой оси $z_{k}=i \operatorname{Im} z_{k}, 0<$ $\operatorname{Im} z_{k}<1$, таких, что $\operatorname{Im} z_{k} \uparrow 1$ и $V\left(z_{k}\right)=V(i)$; при этом

$$
V\left(\bar{z}_{k}\right)=V\left(-z_{k}\right)=\overline{V(i)}
$$

K дальнейшим свойствам функции Павлова мы будем обрашаться по мере необходимости. Положим

$$
f(\lambda)=-\frac{1}{V(-z(\lambda))}, \quad \lambda(z)=\frac{z+z^{-1}}{2} .
$$

Ясно, что функция $f$ голоморфна в $\{\operatorname{Im} \lambda>0\}$ и непрерывна (на самом деле бесконечно дифференцируема) в $\{\operatorname{Im} \lambda \geqslant 0\}$, причем $\operatorname{Im} f(x)=0$ при $x \in(-\infty,-1] \cup$ $[1, \infty)$ и $\operatorname{Im} f(x)>0$ при $\lambda \in\{\operatorname{Im} \lambda>0\} \cup(-1,1)$. Следовательно,

$$
f(\lambda)=\alpha \lambda+\beta+\int_{-1}^{1} \frac{\operatorname{Im} f(x)}{x-\lambda} d x, \quad \alpha \geqslant 0, \quad \beta=\bar{\beta} .
$$

Коэффициент $\alpha$ находится из предельного соотношения

$$
\alpha=\lim _{y \rightarrow+\infty} \frac{f(i y)}{i y}=-\lim _{t \downarrow 0} \frac{2}{i\left(t^{-1}-t\right) V(i t)},
$$

и поскольку $V(i t)=V^{\prime}(0) i t+O\left(t^{2}\right)$ при $t \rightarrow 0$, то

$$
\alpha=\frac{2}{V^{\prime}(0)}=\frac{2 e^{1 / 32}}{\cos \gamma}>0 .
$$

Определим

$$
\widetilde{m}(\lambda):=A \int_{-1}^{1} \frac{\operatorname{Im} f(x)}{x-\lambda} d x=A(f(\lambda)-\alpha \lambda-\beta), \quad A^{-1}:=\int_{-1}^{1} \operatorname{Im} f(x) d x .
$$


Функция $\widetilde{m}(\lambda)$ является функцией Вейля некоторой матрицы Якоби

$$
\widetilde{J}=\left(\begin{array}{cccc}
b_{1} & a_{1} & & \\
a_{1} & b_{2} & a_{2} & \\
& \ddots & \ddots & \ddots
\end{array}\right), \quad a_{j}>0, \quad b_{j}=\overline{b_{j}} .
$$

Положим, наконец,

$$
a_{0}^{2}:=-\frac{1}{\alpha A}, \quad b_{0}:=-\frac{\beta}{\alpha}-\frac{1}{\alpha \overline{V(i)}}, \quad a_{0}, b_{0} \in \mathbb{C},
$$

где $\alpha$ и $\beta$ взяты из представления (5.2), и построим комплексную якобиеву матрицу

$$
J=\left(\begin{array}{cc}
b_{0} & a_{0} \\
a_{0} & \widetilde{J}
\end{array}\right)
$$

Нашей целью является доказательство того, что $J$ удовлетворяет условиям теоремы 2 с $\nu=0 .^{1}$

Начнем с дискретного спектра матрицы $J$. Пусть $R(\lambda)=(J-\lambda)^{-1}$ - резольвента $J$ и $m(\lambda)=\left(R(\lambda) e_{0}, e_{0}\right)-$ ее функция Вейля. Как известно [23],

$$
m(\lambda)=\frac{1}{\lambda-b_{0}-a_{0}^{2} \widetilde{m}(\lambda)},
$$

и в силу выбора $a_{0}, b_{0}(5.4)$ и определения $\widetilde{m}$ мы имеем

$$
\begin{aligned}
\lambda-a_{0}^{2} \tilde{m}(\lambda)-b_{0} & =\lambda+\frac{\widetilde{m}(\lambda)}{\alpha A}+\frac{\beta}{\alpha}+\frac{1}{\alpha \overline{V(i)}}=\frac{1}{\alpha}\left(f(\lambda)+\frac{1}{\overline{V(i)}}\right) \\
& =\frac{1}{\alpha}\left(\frac{1}{\overline{V(i)}}-\frac{1}{V(-z(\lambda))}\right) .
\end{aligned}
$$

Пусть $\lambda_{k}=\lambda\left(z_{k}\right)=\frac{1}{2}\left(z_{k}+z_{k}^{-1}\right)$, где $z_{k}$ из іv), так что $\lambda_{k} \in i \mathbb{R}_{-}, \lambda_{k} \rightarrow 0$ при $k \rightarrow \infty$. Свойство iv) функции $V$ влечет $\lambda_{k}-b_{0}-a_{0}^{2} \tilde{m}\left(\lambda_{k}\right)=0$, т.е. $m$ имеет полюсы в точках $\lambda_{k}$, тем самым, $\left\{\lambda_{k}\right\} \in \sigma_{d}(J)$ и 0 есть предельная точка $\sigma_{d}(J)$.

Покажем, что других предельных точек нет. Начнем с простого теоретико-операторного утверждения.

ЛЕмма 5.1. Пусть T - ограниченный оператор в гильбертовом пространстве $\mathscr{H}$ такой, что $T$ и $T^{*}$ имеют общий циклический вектор $h$. Точка $\lambda \in \mathbb{C}$ является изолированной точкой спектра $T$ в том и только том случае, если $\lambda$ есть особая точка функиии Вейля $m(\lambda)=\left(R(\lambda) e_{0}, e_{0}\right), R(\lambda)=(T-\lambda)^{-1}$.

\footnotetext{
${ }^{1}$ На заключительном этапе доказательства мы покажем, что общий случай несущественно отличается от этого специального.
} 
ДоКАЗАТЕЛЬСТво. Надо лишш проверить, что изолированная особенность резольвенты $R(\lambda)$ является в данном случае неустранимой особенностью функции Вейля. Предположим, что $m$ голоморфна в точке $\lambda$. Поскольку

$$
\left(R(\mu) T^{k} h, h\right)=\left(R(\mu)\left(T^{k}-\mu^{k}\right) h, h\right)+\mu^{k} m(\mu)=P(\mu)+\mu^{k} m(\mu),
$$

где $P$ - полином, то функции $\left(R(\mu) g_{n}, h\right)$, где $g_{n}=\sum_{k=0}^{n} c_{k} T^{k} h$, также голоморффны в точке $\lambda$. По условию система $\left\{T^{k} h\right\}_{k} \geqslant 0$ полна в $\mathscr{H}$, так что для любого $g \in \mathscr{H}$ найдется последовательность $g_{n} \rightarrow g$ при $n \rightarrow \infty$. При достаточно малом $\varepsilon>0$ проколотый круг $\{\mu: 0<|\mu-\lambda| \leqslant \varepsilon\}$ целиком лежит в резольвентном множестве оператора $T$, т.е. $\|R(\mu)\| \leqslant C$ при $|\mu-\lambda|=\varepsilon$. Значит, $\left(R(\mu) g_{n}, e_{0}\right)$ сходится к $\left(R(\mu) g, e_{0}\right)$ равномерно в этом круге, так что $\left(R(\mu) g, e_{0}\right)$ голоморфна в точке $\lambda$. Аналогичным образом, учитывая полноту системы $\left\{\left(T^{*}\right)^{k} h\right\}_{k \geqslant 0}$, устанавливается голоморфность функции $(R(\mu) g, f)$ в точке $\lambda$ при всех $g, f \in \mathscr{H}$. Но слабая голоморфность влечет сильную (см., например, [24; гл. V.3]), т.е. $R$ голоморфна в точке $\lambda$, что и требовалось.

Предположим теперь, что сушествует последовательность $\left\{\lambda_{k}^{\prime}\right\} \in \sigma_{d}(J)$ и $\lambda^{\prime}=$ $\lim _{k \rightarrow \infty} \lambda_{k}^{\prime} \in[-1,1]$, причем $\lambda^{\prime} \neq 0$. По лемме 5.1 функция Вейля $m$ имеет полюсы в точках $\lambda_{k}^{\prime}$ и в силу $(5.4),(5.5) V\left(-z\left(\lambda_{k}^{\prime}\right)\right)=\overline{V(i)}$. Но

$$
-\lim _{k \rightarrow \infty} z\left(\lambda_{k}^{\prime}\right)=y^{\prime} \in \mathbb{T} \backslash\{ \pm i\}
$$

и поскольку функция $V$ голоморфна в точке $y^{\prime}$, то $V \equiv \overline{V(i)}$, что противоречит определению функции Павлова $V$ (5.1).

Основная трудность состоит в доказательстве того, что матрища $J$ принадлежит классу $\mathscr{P}(1 / 2-\varepsilon)$. В качестве первого шага установим некоторую предварительную оценку скорости стабилизации параметров $\left\{a_{n}, b_{n}\right\}$, которые суть коэффициенты трехчленного рекуррентного соотношения для ортонормированных полиномов относительно веса $w(x)=A \operatorname{Im} f(x)$ на $[-1,1]$, где постоянная $A$ определена формулой (5.3). Поскольку функция Павлова $V$ голоморфна в 1 и $V(1)>0$, $V^{\prime}(1)>0$, то

$$
\operatorname{Im} V\left(e^{i \theta}\right)=V^{\prime}(1) \sin \theta+O\left(\sin ^{2} \theta\right)
$$

при $\theta$ вблизи $0, \pi$, и функция

$$
\frac{w(\cos \theta)}{\sin \theta}=\frac{\operatorname{Im} V\left(e^{i \theta}\right)}{\sin \theta\left|V\left(e^{i \theta}\right)\right|^{2}}, \quad 0 \leqslant \theta \leqslant \pi,
$$

является $2 \pi$-периодической и бесконечно дифференцируемой. Следовательно, для ее коэффициентов Фурье справедливо

$$
\frac{w(\cos \theta)}{\sin \theta}=\sum_{n=-\infty}^{\infty} q_{n} e^{i n \theta}, \quad \sum_{n=1}^{\infty} n^{k}\left|q_{n}\right|<\infty
$$


для любого натурального $k$. По [25; теорема 1] такое же неравенство имеет место и для параметров $\left\{a_{n}, b_{n}\right\}$ :

$$
\sum_{n=1}^{\infty} n^{k}\left(\left|a_{n}-\frac{1}{2}\right|+\left|b_{n}\right|\right)<\infty, \quad k=1,2, \ldots
$$

Соотношения (5.7) позволяют применить технику задачи рассеяния, развитую в предыдушем параграфе. Заметим, что это не просто “дань традиции”, а адекватный аппарат, позволяющий получить правильную оценку убывания элементов $a_{n}-1 / 2$ и $b_{n}$ для веса, связанного с функцией Павлова. Мы можем лишш надеяться, что современные методы (задача Римана-Гильберта) позволят решить данную задачу в более общем классе весов столь же эффективно, как это сделано для весов, аналитических ${ }^{2}$ в окрестности $[-1,1]$ (см. [26]).

Пусть $\left\{\widetilde{f}_{n}\right\}_{n \geqslant 0}$ - решение Йоста уравнения $(\widetilde{J}-\lambda) y=0$. Так как это решение с точностью до мультипликативной постоянной, зависящей от спектрального параметра, совпадает с решением Вейля того же уравнения, то

$$
\widetilde{M}(z):=-\widetilde{m}(\lambda(z))=\frac{\widetilde{f}_{1}(z)}{\widetilde{f}_{0}(z)},
$$

откуда с использованием выражения (4.3) для вронскиана $\langle\widetilde{f}, \overline{\widetilde{f}}\rangle$ получаем

$$
\left|\widetilde{f}_{0}\left(e^{i \theta}\right)\right|^{2}=\left|\frac{\sin \theta}{\operatorname{Im} \widetilde{M}\left(e^{i \theta}\right)}\right| .
$$

Начнем с того, что установим отсутствие резонансов в спектре матрицы $\widetilde{J}$. Предположим, напротив, что $\widetilde{f}_{0}( \pm 1)=0$ хотя бы при одном выборе знака. Так как функция $A^{-1} \widetilde{m}(\lambda)=-V^{-1}(-z(\lambda))-\alpha \lambda-\beta$ ограничена в окрестности точек $\lambda= \pm 1$, то из $(5.8)$ следует $\widetilde{f}_{1}( \pm 1)=0$ и, значит, в силу рекуррентного соотношения $(4.2)$ $\widetilde{f}_{n}( \pm 1)=0$, что противоречит асимптотике решения Йоста.

Помимо указанных выше свойств i)-iv) функции Павлова в [2; формула (3.1)] найдены оценки ее производных

$$
\left|V^{(r)}\left(e^{i \theta}\right)\right| \leqslant K^{r} r ! r^{r / \delta}, \quad \delta=1-\gamma, \quad r=0,1,2, \ldots,
$$

где $K=K(\gamma)$ - некоторая положительная константа. Мы покажем, что функция рассеяния $\widetilde{S}=\widetilde{\widetilde{f}}_{0} \widetilde{f}_{0}^{-1}$ удовлетворяет тем же оценкам, возможно, с некоторой другой константой $K$. Согласно [2; лемма $3 . П .1$ и замечание], если для функции $v$ справедливо (5.10), а функции $u$ и $u_{1}$ голоморфны в окрестности множества значений $v$ и замкнутого круга $\overline{\mathbb{D}}$ соответственно, то сложная функция $u(v)$ и произведение $u_{1} v$ удовлетворяют (5.10) (с некоторой другой постоянной $K$ ). Поэтому функция Вейля

$$
\widetilde{M}(z)=A\left(\frac{1}{V(-z)}+\frac{\alpha}{2}\left(z+z^{-1}\right)+\beta\right)
$$

\footnotetext{
${ }^{2}$ Наш вес $w$ имеет одну особую точку в нуле.
} 
удовлетворяет (5.10) $\left(V\left(e^{i \theta}\right) \neq 0\right)$. Далее, так как в силу (5.6)

$$
\left|\frac{\sin \theta}{\operatorname{Im} \widetilde{M}\left(e^{i \theta}\right)}\right| \geqslant C>0
$$

при всех $\theta$, то с учетом (5.9) мы имеем

$$
\left|\left(\ln \left|\widetilde{f}_{0}\left(e^{i \theta}\right)\right|\right)^{(r)}\right| \leqslant K_{1}^{r} r ! r^{r / \delta}, \quad K_{1}=K_{1}(\gamma)>0, \quad r \in \mathbb{Z}_{+} .
$$

Для функции рассеяния $\widetilde{S}$ справедливо

$$
\widetilde{S}\left(e^{i \theta}\right)=\exp \left(-2 i \arg \widetilde{f}_{0}\left(e^{i \theta}\right)\right)=\exp \left(-2 i H\left(\ln \left|\widetilde{f}_{0}\right|\right)\left(e^{i \theta}\right)\right),
$$

так что остается проверить, что класс функций (5.10) инвариантен относительно оператора Гильберта $H$.

ЛЕмма 5.2. Пусть $g-2 \pi$-периодическая и бесконечно дифференцируемая функиия. Тогда при любом натуральном $n$

$$
\left\|(H g)^{(n)}\right\|_{\infty} \leqslant C\left(\left\|g^{(n)}\right\|_{\infty}+\left\|g^{(n+1)}\right\|_{\infty}\right),
$$

где С - абсолютная постоянная, а оператор Гильберта определяется равенством

$$
(H g)(\theta)=\frac{1}{\pi} \int_{0}^{\pi} \frac{g(\theta-t)-g(\theta+t)}{2 \operatorname{tg}(t / 2)} d t .
$$

ДОКАЗАТЕЛЬСТВО. В условиях леммы интеграл (5.11) абсолютно сходится и оператор $H$ перестановочен с дифференцированием $(H g)^{\prime}=H g^{\prime}$. Далее, для функции $g$ имеет место представление

$$
g(x)=\frac{1}{\pi} \int_{-\pi}^{\pi} g(x) d x+\frac{1}{\pi} \int_{-\pi}^{\pi} \varphi(t) g^{\prime}(x+t) d t
$$

где

$$
\varphi(t)= \begin{cases}\frac{t-\pi}{2}, & 0<t \leqslant \pi \\ \frac{t+\pi}{2}, & -\pi \leqslant t<0\end{cases}
$$

которое получается интегрированием по частям в частной сумме ряда Фурье и предельным переходом. Из этого представления получаем

$$
\|g\|_{\infty} \leqslant C\left(\|g\|_{2}+\left\|g^{\prime}\right\|_{2}\right)
$$

Поскольку оператор Гильберта унитарен в $L^{2}(\mathbb{T})$, мы имеем

$$
\|H g\|_{\infty} \leqslant C\left(\|g\|_{2}+\left\|g^{\prime}\right\|_{2}\right) \leqslant C\left(\|g\|_{\infty}+\left\|g^{\prime}\right\|_{\infty}\right),
$$

что и требовалось доказать. 
Таким образом, для функции рассеяния выполняется

$$
\left|\widetilde{S}^{(r-1)}\left(e^{i \theta}\right)\right| \leqslant K_{2}^{r} r ! r^{r / \delta}, \quad K_{2}=K_{2}(\gamma)>0, \quad r=1,2, \ldots
$$

Переход к матричным элементам $a_{n}, b_{n}$ осушествляется с помошью леммы 4.2. Проинтегрируем $r$ раз по частям представление (4.7) с учетом периодичности функции $\widetilde{S}$. Тогда

$$
\begin{gathered}
n^{r}(|F(2 n+2)-F(2 n)|+|F(2 n-1)-F(2 n+1)|) \\
\leqslant \frac{1}{2 \pi 2^{r}} \int_{0}^{2 \pi}\left|\frac{d^{r}}{d \theta^{r}}\left(\widetilde{S}\left(e^{i \theta}\right)\left(e^{2 i \theta}-1\right)\right)\right| d \theta .
\end{gathered}
$$

Так как умножение на регулярную функцию не меняет структуры оценок (5.12), то (с некоторой положительной постоянной $K_{3}$ )

$$
|F(2 n+2)-F(2 n)|+|F(2 n-1)-F(2 n+1)| \leqslant C K_{3}^{r} r ! r^{r / \delta} n^{-r+1} \leqslant C n \inf _{r \geqslant 3} t^{r} r^{\alpha r},
$$

где $t=K_{3} n^{-1} \ll 1$ и $\alpha=1+1 / \delta$. Применим к правой части неравенства $(5.13)$ оценку (3.6). Для больших $n$ имеем

$$
|F(2 n+2)-F(2 n)|+|F(2 n-1)-F(2 n+1)| \leqslant C \exp \left(-C_{1} n^{\delta /(1+\delta)}\right),
$$

где

$$
C_{1}=\frac{1+\delta}{2 \delta} K_{3}^{-\delta /(1+\delta)}>0
$$

Из (4.10) и (5.14) вытекает оценка

$$
\widehat{F}^{2}(2 n-2) \leqslant C \exp \left(-C_{1} n^{\delta /(1+\delta)}\right) \sum_{k=n}^{\infty} \exp \left(-C_{1} k^{\delta /(1+\delta)}\right) \leqslant C_{2} \exp \left(-C_{1} n^{\delta /(1+\delta)}\right)
$$

и в силу леммы 4.2 соответственно

$$
\left|a_{n}-\frac{1}{2}\right|+\left|b_{n}\right| \leqslant C_{3} \exp \left(-C_{4} n^{\delta /(1+\delta)}\right)=C_{3} \exp \left(-C_{4} n^{(1-\gamma) /(2-\gamma)}\right), \quad C_{3}, C_{4}>0 .
$$

При заданном $\varepsilon>0$ остается выбрать $\gamma$ столь малым, чтобы

$$
\frac{1-\gamma}{2-\gamma}>\frac{1}{2}-\varepsilon
$$

что и означает $\widetilde{J} \in \mathscr{P}\left(\frac{1}{2}-\varepsilon\right)$. Теорема 2 тем самым доказана при $\nu=0$.

Общий случай получается с помощью тех же рассуждений с использованием модифицированной функции Павлова. Действительно, пусть $-1<\varkappa<1$. Рассмотрим функцию

$$
V_{\varkappa}(z)=V\left(\frac{z-\varkappa}{1-\varkappa z}\right), \quad V_{0}(z)=V(z) .
$$

Эта функция удовлетворяет i)-iv) с той лишш разницей, что ее особыми точками на $\mathbb{T}$ являются точки $w_{\varkappa}$ и $\bar{w}_{\varkappa}$, где $w_{\varkappa}=(i+\varkappa)(1+i \varkappa)^{-1}$. Когда $\varkappa$ пробегает интервал $(-1,1)$, точка $w_{\varkappa}$ описывает верхнюю полуокружность. Таким образом, при подходящем выборе $\varkappa$ мы получим произвольную точку $\nu,-1<\nu<1$, сгущения дискретного спектра якобиевой матрицы $J \in \mathscr{P}(1 / 2-\varepsilon)$. Теорема 2 полностью доказана. 


\section{§6. Приложение}

Мы докажем здесь два утверждения об определителях возмушения комплексных якобиевых матриц, следуя [6; раздел 2], где эти утверждения доказаны для вещественных симметрических матрич.

ДОКАЗАТЕЛЬСТВО УТВЕРЖДЕНИЯ 2.2. Мы использУем известные свойства операторных идеалов Шаттена-фон Неймана $\mathscr{S}_{p}$ и бесконечных определителей (см., например, [5; гл. III, IV]). Доказательство распадается на несколько этапов.

1) Пусть

$$
R_{0}(z)=\left(J_{0}-\lambda(z)\right)^{-1}, \quad R_{0}(z)=\left\{r_{n m}(z)\right\}_{0}^{\infty}, \quad|z|<1,
$$

- резольвента дискретного лапласиана. Для матричных элементов $r_{n m}$ непосредственно находится

$$
r_{n m}(z)=\frac{z^{|n-m|}-z^{n+m+2}}{z-z^{-1}}=-\sum_{j=0}^{\min \{n, m\}} z^{1+|n-m|+2 j}, \quad n, m \geqslant 0, \quad|z|<1 .
$$

Ясно, что

$$
\left|r_{n m}(z)\right| \leqslant \min \{n, m\}+1 .
$$

Пусть $|\zeta|=1$. Очевидно, существует предельное значение $r_{n m}(\zeta)$, однако матрица $\left\{r_{n m}(\zeta)\right\}$ определена лишь формально (ей не отвечает ограниченньй оператор в $\left.\ell^{2}\right)$. Ситуацию можно исправить путем окаймления $R_{0}$ диагональными матрицами. Пусть

$$
D=\operatorname{diag}\left(d_{0}, d_{1}, \ldots\right), \quad d_{j} \geqslant 0, \quad \sum_{j} j d_{j}<\infty .
$$

Рассмотрим оператор-функцию

$$
B(z):=D^{1 / 2} R_{0}(z) D^{1 / 2}=\left\{B_{n m}(z)\right\}_{0}^{\infty}, \quad|z|<1 .
$$

Так как $D^{1 / 2} \in \mathscr{I}_{2}$ (оператор Гильберта-Шмидта), то $B(z) \in \mathscr{S}_{2}$ (на самом деле $B \in \mathscr{S}_{1}$ как произведение двух операторов из $S_{2}$, чем мы воспользуемся далее). Более того, по (6.1)

$$
\|B(z)\|_{2}^{2}=\sum_{n, m}\left|B_{n m}(z)\right|^{2} \leqslant \sum_{n, m}\left(d_{m}^{1 / 2} d_{n}^{1 / 2}(\min \{n, m\}+1)\right)^{2} \leqslant\left(\sum_{j}(j+1) d_{j}\right)^{2}
$$

и тем самым предельные значения $B(\zeta)=\lim _{z \rightarrow \zeta} B(z)$ принадлежат $\mathscr{S}_{2}$ и удовлетворяют (6.2). Наконец,

$$
\|B(z)-B(\zeta)\|_{2}^{2}=\sum_{n, m}\left|B_{n m}(z)-B_{n m}(\zeta)\right|^{2} \rightarrow 0, \quad z \rightarrow \zeta,
$$

по теореме Лебега о мажорированной сходимости, так что $B(z)$ принадлежит алгебре $\mathscr{A}$ (как оператор-функция со значениями в $\left.\mathscr{S}_{2}\right)$. 
Пусть теперь $J$ - якобиева матрища (1.2), подчиненная условию (1.3). Положим $d_{0}=\max \left\{\left|b_{0}\right|,\left|c_{0}-1 / 2\right|\right\}, d_{n}=\max \left\{\left|a_{n-1}-1 / 2\right|,\left|b_{n}\right|,\left|c_{n}-1 / 2\right|\right\}, n \geqslant 1$ и и $D=\operatorname{diag}\left(d_{0}, d_{1}, \ldots\right)$. Возмущение $\Delta J=J-J_{0}$ допускает факторизацию $\Delta J=$ $D^{1 / 2} U D^{1 / 2}$, где

$$
U=\left\{u_{n m}\right\}, \quad u_{n m}= \begin{cases}\frac{a_{n-1}-1 / 2}{\sqrt{d_{n-1} d_{n}}}, & m=n-1 ; \\ \frac{b_{n}}{d_{n}}, & m=n ; \quad u_{n m}=0, \quad|n-m| \geqslant 2, \\ \frac{c_{n}-1 / 2}{\sqrt{d_{n+1} d_{n}}}, & m=n+1,\end{cases}
$$

в котором считаем $0 / 0=1$. Очевидно, $\left|u_{n m}\right| \leqslant 1$, так что $\|U\| \leqslant 3$.

2) Рассмотрим функцию $t(z):=\operatorname{tr}\left(\Delta J R_{0}(z)\right)$, корректно определенную при $|z|<1$ при условии $\Delta J \in \mathscr{S}_{1}$. Покажем, что $(2.1)$ влечет $t \in \mathscr{A}$, и получим оценку сверху для этой функции. По определению следа $t=t_{1}+t_{2}+t_{3}$, где

$$
\begin{gathered}
t_{1}(z)=\sum_{0}^{\infty} b_{j} r_{j j}(z), \quad t_{2}(z)=\sum_{0}^{\infty}\left(a_{j}-\frac{1}{2}\right) r_{j, j+1}(z) \\
t_{3}(z)=\sum_{0}^{\infty}\left(c_{j}-\frac{1}{2}\right) r_{j+1, j}(z)
\end{gathered}
$$

Неравенство (6.1) и условие (2.1) позволяют, как и выше, заключить, что $t_{l} \in \mathscr{A}$, $l=1,2,3$, и

$$
|t(z)| \leqslant \sum_{0}^{\infty}(j+2)\left(\left|a_{j}-\frac{1}{2}\right|+\left|b_{j}\right|+\left|c_{j}-\frac{1}{2}\right|\right) .
$$

3) Запишем определитель возмушения $\Delta$ с учетом факторизации $\Delta J$ :

$$
\Delta(z, J)=\operatorname{det}\left(I+\Delta J R_{0}\right)=\operatorname{det}\left(I+D^{1 / 2} U D^{1 / 2} R_{0}\right)=\operatorname{det}(I+U B(z))
$$

(здесь использовано свойство $\operatorname{det}(I+A B)=\operatorname{det}(I+B A)$ ). Поскольку $B(z)$ имеет "хорошие" свойства как функция со значениями в $\mathscr{S}_{2}$, естественно перейти к регуляризованным определителям [5; гл. IV,$\S 2]$ :

$$
\Delta(z, J)=\operatorname{det}_{2}(I+U B(z)) e^{\operatorname{tr}\left(\Delta J R_{0}(z)\right)} .
$$

В силу непрерывности $\operatorname{det}_{2}$ по операторному аргументу [4; теорема IV .2.1] и непрерывности $t$ в $\overline{\mathbb{D}}$ из (6.4) следует $\Delta \in \mathscr{A}$.

Запишем теперь (6.4) для ассоциированной матрицы $J^{(k)}$ :

$$
\Delta\left(z, J^{(k)}\right)=\operatorname{det}_{2}\left(I+U_{k} B_{k}(z)\right) e^{\operatorname{tr}\left(\Delta J^{(k)} R_{0}(z)\right)} .
$$


Окаймляющая матрища $D_{k}$ сейчас имеет вид $D_{k}=\operatorname{diag}\left(d_{0}^{(k)}, d_{1}^{(k)}, \ldots\right)$, где $d_{j}^{(k)}=$ $d_{j+k}$. В силу (6.2)

$$
\left\|B_{k}(z)\right\|_{2}^{2} \leqslant\left(\sum_{j}(j+1) d_{j}^{(k)}\right)^{2}
$$

и по теореме Лебега $\lim _{k \rightarrow \infty}\left\|B_{k}(z)\right\|_{2}=0$ равномерно в $\overline{\mathbb{D}}$, откуда следует

$$
\lim _{k \rightarrow \infty} \operatorname{det}_{2}\left(I+U_{k} B_{k}(z)\right)=1
$$

В силу (6.3)

$$
\left|t^{(k)}(z)\right|=\left|\operatorname{tr}\left(\Delta J^{(k)} R_{0}(z)\right)\right| \leqslant \sum_{0}^{\infty}(j+2)\left(\left|a_{j+k}-\frac{1}{2}\right|+\left|b_{j+k}\right|+\left|c_{j+k}-\frac{1}{2}\right|\right),
$$

так что $\lim _{k \rightarrow \infty}\left|t^{(k)}(z)\right|=0$ равномерно в $\overline{\mathbb{D}}$, и утверждение доказано.

ДОКАЗАТЕЛЬСТВО ПРЕДЕЛЬНОГО СООТНОШЕНИЯ (2.2). Идея состоИт в сЛедуюшем: необходимо аппроксимировать оператор $\Delta J R_{0}$ конечномерными операторами и учесть непрерывность определителя возмущения.

Пусть

$$
\widehat{J}_{m}:=\left(\begin{array}{rr}
J_{m} & \vdots \\
\cdots \cdots & \ldots \\
\vdots & J_{0}
\end{array}\right), \quad \widehat{J}_{0, m}:=\left(\begin{array}{rr}
J_{0, m} & \vdots \\
\cdots \cdots & \ldots \\
\vdots & J_{0}
\end{array}\right)
$$

- блочно-диагональные операторы,

$$
\Delta \widehat{J}_{m}=\widehat{J}_{m}-\widehat{J}_{0, m}=\left(\begin{array}{r}
J_{m}-J_{0, m} \\
\cdots \cdots \cdots \cdots \\
\vdots \\
\cdots
\end{array}\right) .
$$

Поскольку

$$
\Delta J=J-J_{0}=\left(\begin{array}{ccc}
J_{m}-J_{0, m} & \vdots & C \\
\ldots \ldots \ldots \ldots \ldots \ldots & \ldots \ldots \ldots \\
A & \vdots & J^{(m)}-J_{0}
\end{array}\right),
$$

где матрицы $A$ и $C$ имеют только по одному ненулевому элементу $C_{m 1}=c_{m}-1 / 2$, $A_{1 m}=a_{m}-1 / 2, A_{j i}=C_{i j}=0, i \neq m, j \neq 1$, то

$$
\left\|\Delta J-\Delta \widehat{J}_{m}\right\|_{1} \leqslant \sum_{j=m}^{\infty}\left(\left|a_{j}-\frac{1}{2}\right|+\left|b_{j}\right|+\left|c_{j}-\frac{1}{2}\right|\right) \rightarrow 0, \quad m \rightarrow \infty .
$$

Далее, как легко видеть, матрица $J_{0}-\widehat{J}_{0, m}$ имеет лишь два ненулевых элемента. Если $h \in \ell^{2}$, то

$$
\left(J_{0}-\widehat{J}_{0, m}\right) h=\left(h, e_{m}\right) e_{m+1}+\left(h, e_{m+1}\right) e_{m} \rightarrow 0, \quad m \rightarrow \infty,
$$


иными словами, $\widehat{J}_{0, m} \rightarrow J_{0}$ при $m \rightarrow \infty$ в сильном смысле (на каждом векторе из $\ell^{2}$ ). Пусть $K-$ компакт, лежащий в $\mathbb{D}$. Сравним резольвенты операторов $J_{0}$ и $\widehat{J}_{0, m}$ при $z \in K$ :

$$
\left(\widehat{J}_{0, m}-\lambda\right)^{-1}-\left(J_{0}-\lambda\right)^{-1}=\left(\widehat{J}_{0, m}-\lambda\right)^{-1}\left(J_{0}-\widehat{J}_{0, m}\right)\left(J_{0}-\lambda\right)^{-1} .
$$

Так как $\left\|\left(\widehat{J}_{0, m}-\lambda\right)^{-1}\right\|=\left(\operatorname{dist}\left(\lambda(z), \sigma\left(\widehat{J}_{0, m}\right)\right)\right)^{-1}$ и $\sigma\left(\widehat{J}_{0, m}\right)=[-1,1]$, то

$$
\left\|\left(\widehat{J}_{0, m}-\lambda\right)^{-1}\right\| \leqslant C(K), \quad z \in K
$$

Следовательно, $\left(\widehat{J}_{0, m}-\lambda\right)^{-1} \rightarrow\left(J_{0}-\lambda\right)^{-1}$ в сильном смысле.

Согласно [5; теорема III.6.3] из сходимости последовательности операторов $A_{m}$ к $A$ в $\mathscr{S}_{1}$ и $X_{m}$ к $X$ в сильном смысле следует сходимость $A_{m} X_{m}$ к $A X$ в $\mathscr{S}_{1}$. Тем самьгм

$\left(\widehat{J}_{m}-\widehat{J}_{0, m}\right)\left(\widehat{J}_{0, m}-\lambda\right)^{-1} \rightarrow \Delta J R_{0}, \quad I+\left(\widehat{J}_{m}-\widehat{J}_{0, m}\right)\left(\widehat{J}_{0, m}-\lambda\right)^{-1} \rightarrow I+\Delta J R_{0}$

в $\mathscr{S}_{1}$ при каждом $z$, а значит, равномерно на $K$. Но $\left(\widehat{J}_{m}-\widehat{J}_{0, m}\right)\left(\widehat{J}_{0, m}-\lambda\right)^{-1}-$ конечномерньй оператор и

$$
\operatorname{det}\left(I+\left(\widehat{J}_{m}-\widehat{J}_{0, m}\right)\left(\widehat{J}_{0, m}-\lambda\right)^{-1}\right)=\operatorname{det}\left(\frac{J_{m}-\lambda}{J_{0, m}-\lambda}\right)
$$

так что остается учесть непрерывность определителя возмущения.

\section{Список литературы}

1. Павлов Б. С. О несамосопряженном операторе Шрёдингера. I // Проблемы математической физики. №1. Л.: Изд-во ЛГУ, 1966. С. 102-132.

2. Павлов Б. С. О несамосопряженном операторе Шрёдингера. II // Проблемы математической физики. № 2. Л.: Изд-во ЛГУ, 1967. С. 133-157.

3. Bairamov E., Cakar O., Krall A.M. Non-selfadjoint difference operators and Jacobi matrices with spectral singularities // Math. Nachr. 2001. V. 229. P. 5-14.

4. Geronimo J. S., Case K.M. Scattering theory and polynomials on the real line // Trans. Amer. Math. Soc. 1980. V. 258. P. 467-494.

5. Гохберг И. Ц., Крейн М. Г. Введение в теорию линейных несамосопряженных операторов в гильбертовом пространстве. М.: Наука, 1965.

6. Killip R., Simon B. Sum rules for Jacobi matrices and their applications to spectral theory // Ann. Math. 2003. V. 158. P. 253-321.

7. Carleson L. Sets of uniqueness for functions analytic in the unit disc // Acta Math. 1952. V. 87. P. 325-345.

8. Aptekarev A.I., Kalyaguine V., Van Assche $W$. Criterion for the resolvent set of nonsymmetric tridiagonal operators // Proc. Amer. Math. Soc. 1995. V. 123. P. 2423-2430.

9. Beckermann $B$. On the convergence of bounded J-fractions on the resolvent set of the corresponding second order difference operator // J. Approx. Theory. 1999. V. 99. P. 369-408.

10. Beckermann B., Kaliaguine $V$. The diagonal of the Padé table and the approximation of the Weyl function of the second order difference operators // Constr. Approx. 1997. V. 13. P. 481-510.

11. Beckermann B. Complex Jacobi matrices // J. Comput. Appl. Math. 2001. V. 127. P. 17-65. 
12. Барриос Д., Лопес Г., Мартинес-Финкельитейн А., Торрано Э. Конечномерные аппроксимации резольвенты бесконечной ленточной матрицы и непрерьвные дроби // Матем. сб. 1999. Т. 190. № 4. С. 23-42.

13. Гусейнов Г. Ш. Определение бесконечной матрицы Якоби по данњым рассеяния // Докл. АН СССР. 1976. Т. 227. №6. С. 1289-1292.

14. Teschl G. Jacobi operators and completely integrable nonlinear lattices. Providence, RI: Amer. Math. Soc., 1999. (Math. Surveys Monogr. V. 72.)

15. Fatou P. Séries trigonométriques et séries de Taylor // Acta Math. 1906. V. 30. P. 335-400.

16. Beurling A. Ensembles exceptionneles // Acta Math. 1939. V. 72. P. 1-13.

17. Хрущев C. В. Проблема одновременной аппроксимации и стирание особенностей интегралов типа Коши // Труды МИАН СССР. 1978. Т. 130. С. 124-195.

18. Besicovitch A.S., Taylor S.J. On the complementary intervals of a linear closed sets of zero Lebesque measure // J. London Math. Soc. 1954. V. 29. P. 449-459.

19. Taylor B. A., Williams D. L. Boundary zero sets of $A^{\infty}$ functions satisfying growth conditions // Proc. Amer. Math. Soc. 1972. V. 35. P. 155-160.

20. Коренблюм Б. И. Квазианалитические классы функций в круге // Докл. АН СССР. 1965. T. 164 . C. $36-39$.

21. Никишин E. M. Дискретный оператор Штурма-Лиувилля и некоторые задачи теории функций // Труды сем. им. И.Г. Петровского. № 10. М.: Изд-во МГУ, 1984. С. 3-77.

22. Тода М. Теория нелинейных решеток. М.: Мир, 1984.

23. Калягин В.A. О рациональных аппроксимациях резольвентной функции разностного оператора второго порядка // УМН. 1994. Т. 49. № 3. С. 181-182.

24. Иосида K. Функциональньй анализ. М.: Мир, 1967.

25. Geronimo J.S. A relation between the coefficients in the recurrence formula and the spectral function for orthogonal polynomials // Trans. Amer. Math. Soc. 1980. V. 260. № 1 . P. $65-82$.

26. Kuijlaars A., McLaughlin K.T.-R., Van Assche W., Vanlessen M. The Riemann-Hilbert approach to strong asymptotics for orthogonal polynomials on $[-1,1] / /$ Adv. Math. 2004. V. 188. P. 337-398.

Физико-технический институт

Поступила в редакцию

низких температур НАН Украины,

02.02 .2004

г. Харьков, Украина

E-mail: golinskii@ilt.kharkov.ua

egorova@ilt.kharkov.ua 